\title{
Monsieur Alexandre : vers un partage du patrimoine mondial
}

\section{Emmanuelle Le Pichon-Vorstman}

\section{Q OpenEdition \\ 1 Journals}

\section{Édition électronique}

URL : https://journals.openedition.org/dhfles/3498

DOI : $10.4000 /$ dhfles.3498

ISSN : 2221-4038

\section{Éditeur}

Société Internationale pour l'Histoire du Français Langue Étrangère ou Seconde

\section{Édition imprimée}

Date de publication : 1 décembre 2012

Pagination : 219-232

ISSN : 0992-7654

\section{Référence électronique}

Emmanuelle Le Pichon-Vorstman, « Monsieur Alexandre : vers un partage du patrimoine mondial »,

Documents pour l'histoire du français langue étrangère ou seconde [En ligne], 49 | 2012, mis en ligne le 05 juillet 2016, consulté le 28 mai 2021. URL : http://journals.openedition.org/dhfles/3498 ; DOI : https:// doi.org/10.4000/dhfles.3498

Ce document a été généré automatiquement le 28 mai 2021.

(C) SIHFLES 


\title{
Monsieur Alexandre : vers un partage du patrimoine mondial
}

\author{
Emmanuelle Le Pichon-Vorstman
}

En 1864 disparaissait Alexandre Vattemare, tour à tour ventriloque et acteur célébré dans l'Europe entière. Dans un recueil traitant de l'histoire de la diffusion de la langue et de la culture française, pourquoi consacrer un article à Alexandre Vattemare ? Cet homme que l'on n'hésite pas à qualifier d'« ambassadeur extravagant», de "pionnier des échanges culturels internationaux » (Tiliette \& Havens, 2007), né au moment où le français perd du terrain en Europe, n'a, à ma connaissance, jamais exprimé le souhait de diffuser la langue et la culture françaises en particulier. Au contraire, la démarche qui l'animait l'a amené d'abord à s'intéresser aux langues des autres, dans un premier lieu pour les rencontrer, puis pour vendre ses spectacles et enfin, pour devenir un passeur culturel. Ce serait un lieu commun de dire qu'apprendre la langue de l'autre, c'est aussi entrer dans une communauté sociale, artistique, politique, littéraire, religieuse plus ou moins éloignée qui petit à petit et immanquablement, entraîne l'individu dans une transformation identitaire (voir par exemple Kramsch, 2008). Mais aujourd'hui, la globalisation produit un flou qui ne cesse de grandir entre les frontières des groupes linguistiques et culturels. Ce flou remet alors en cause non seulement les identités individuelles mais aussi celles des groupes plus ou moins légitimement constitués. Langues et cultures étrangères deviennent à la fois attirantes et menaçantes. On parle alors de sauvegarde, de diffusion, de défense. Chacun s'accroche alors à ce qui représente le mieux le groupe auquel il s'identifie et cherche à faire (re)connaître sa langue et sa culture. S'agit-il ainsi de lutter contre l'influence grandissante de langues et de cultures que l'on considère comme rivales? C'est pour tâcher d'apporter des éléments de réponse qu'il est intéressant d'étudier le parcours de médiateur interculturel d'Alexandre Vattemare. 


\section{Monsieur Alexandre}

2 Le goût d'Alexandre pour le patrimoine culturel est né d'abord d'un formidable don. Né à Paris le 8 novembre 1796, au cœur des révolutions et Guerres napoléoniennes, il grandit en Normandie et découvre rapidement sa ventriloquie. Dès son plus jeune âge, comme il le raconte lui-même dans ses mémoires, il commence ses farces plus ou moins appréciées de ses victimes (Dargent, $\mathrm{AF}^{1}: 31$ ), où raconte-t-il, « il avait pris l'habitude de tromper sa famille en imitant la voix du facteur» (Vattemare, AF : 74). Recalé du séminaire, il part alors pour Paris où il commence ses études pour devenir chirurgien à l'hôpital Saint Louis (Vattemare, AF : 76). Mais très vite, il se fait à nouveau remarquer par ses farces : il fait parler les morts, entraîne la fille d'un professeur avec lui tout en faisant croire à son père qu'il est appelé ailleurs, par exemple. La faculté décide de l'expulser. Il se propose alors au service des soldats français atteints du choléra lorsque l'épidémie tombe sur Paris en 1814. Ainsi, il obtient de raccompagner à Berlin trois cents militaires prussiens malades. Premier voyage hors des frontières de France pendant lequel il sème ses mystifications, au grand dam des locaux. À plusieurs reprises, il doit s'enfuir au petit matin, de peur de subir les représailles des habitants dont il s'est joué. Il conduit enfin les soldats convalescents à Berlin, où il n'est pas autorisé à exercer la médecine. Il doit alors, pour la première fois, exercer ses talents de ventriloque pour gagner sa vie, suivant en cela les conseils de la famille d'émigrés français qui l'a recueilli à Berlin et dont il finit par épouser la fille. C'est alors que commence pour lui une carrière d'acteur qui l'amènera à jouer dans toutes les villes de l'Europe centrale où il remporte rapidement un succès immense.

\section{L'ambassadeur de la langue française est d'abord polyglotte.}

D'où vient le succès d'Alexandre? Dès le début de son parcours, Alexandre Vattemare a conscience que pour faire rire, il faut présenter aux spectateurs un monde qu'ils reconnaissent. Ainsi, il déplace son regard dans le regard de ceux qui vont l'observer sur la scène. Son don naturel pour la ventriloquie est accompagné d'une formidable aptitude à imiter, puissante stratégie interculturelle. Mais pour imiter, il faut observer avec précision. C'est cette observation participante qui lui permet d'apprendre à connaître ceux qu'il rencontre. Pour cela, il étudie les costumes locaux. Il peut ainsi affiner ses déguisements et ses transformations. Il fouille les bibliothèques, les musées des villes et des châteaux. Il est avide de connaissances.

4 Tout comme l'Europe, Alexandre se transforme pour gagner l'Europe et pour mieux connaître l'autre. Cette transformation passe d'abord par l'apprentissage de la langue de ceux qu'il rencontre. Alexandre apprend donc les langues, indissociables de la culture. De cet apprentissage, il ne dit pas grand-chose, mais on sait par les coupures de journaux qu'il joue déjà en allemand en Allemagne ce qui nous renseigne sur le niveau de langue atteint. Devant le succès qu'il remporte, il continue son périple à travers toute l'Europe. Ami de Goethe, il rapporte qu'il communique avec lui en français mêlé de mots d'allemands. Là encore, stratégie plurilingue par excellence, le changement de code participe d'une connivence née de la connaissance mutuelle des amis. Salué par de nombreuses personnalités dont Thorbecke pour les Pays-Bas, pour être compris et apprécié, il jouait dans la langue du pays, en allemand en Allemagne et en Autriche, 
mais aux Pays-Bas, en Pologne, en Russie, en Hongrie et en Italie jusqu'en 1820, c'est en français qu'il s'exprimait. À Prague, il joue en français et en allemand. En Angleterre où il passe plus de deux années, il joue en anglais. Le français jouit encore d'un rôle prégnant au début du XIX ${ }^{e}$ siècle malgré son déclin engagé (Bruneau 1948). Seul acteur sur la scène, il représente jusqu'à huit personnages de sexe, d'âge, de profession ou de nationalité différentes, apparaissant parfois sous soixante formes différentes (Dargent, AF : 60). Depuis toujours, il imite les voix, mais le fait que différentes nationalités soient représentées dans ses spectacles nous permet de deviner qu'il imite parfaitement les accents et les tournures de phrases. À tel point que l'auditoire en vient à douter de la présence de ce personnage unique sur la scène. En Angleterre, certains journalistes mettent en cause son intégrité. Peu importe: dans les villes où il passe, il reçoit un accueil chaleureux ce qui nous est rapporté par la presse locale sous forme de résumé de la pièce, de description des farces, des costumes et des personnages. Des personnalités célèbres telles que Walter Scott, parmi d'autres spectateurs enthousiastes sur ses talents, lui dédient des poèmes. Au cours de ses voyages, l'auteur note tous les détails des lieux qu'il visite. Il rassemble les objets, articles de journaux, dédicaces et appréciations qu'il reçoit. Souvent, lorsqu'il arrive dans une nouvelle ville, il présente la lettre de recommandation d'un personnage important. Il est important de se souvenir qu'à l'époque, on ne pouvait partir donner un spectacle et revenir le jour suivant. Les voyages s'étalaient sur des semaines, des mois, parfois des années. Alexandre doit rencontrer les locaux pour vendre ses spectacles. Ainsi il est amené à converser avec les puissants, princes, ministres et rois comme à Bruxelles où il est reçu par le prince d'Orange, ainsi que par la reine, princesse d'Orange née Grande Duchesse de Russie, avec qui il s'entretient plus de trois-quarts d'heure (Vattemare, AF : 149).

\section{Plus qu'un polyglotte, un homme avide de rencontres}

5 Pourtant, même s'il était compris par les populations locales, il ne se lassait ni d'apprendre leurs langues, ni de se renseigner sur les us et coutumes des habitants des lieux visités. Alexandre avait compris que seule une connaissance, aussi poussée que possible de la langue indissociable de la culture, lui permettrait de promouvoir ses spectacles. Mais cette curiosité qui pousse Monsieur Alexandre à apprendre les langues des locaux, à comprendre leur rire pour pouvoir mieux le provoquer, ne s'arrête pas aux connaissances des langues, voire des us et coutumes. Pour lui, l'interculturel s'exprime d'abord par la rencontre.

6 Par la découverte de l'étranger qu'il rencontre, il entre dans la vie artistique des pays qu'il parcourt. L'accumulation de connaissances, de marques de reconnaissance sociale et artistique ne lui suffit pas. Alexandre aime celui qu'il rencontre et cela se traduit très concrètement. En parcourant sa vie, en particulier entre 1824 et 1825, on voit qu'il joue pour les puissants mais que son caractère philanthrope l'amène aussi à jouer pour les pauvres, auxquels il distribue une partie de sa fortune. Il joue au profit des aveugles ou des orphelins en Angleterre, des victimes d'un incendie terrible dans telle ou telle ville, et n'attend rien en retour. Jusqu'en 1827, il voyage avec sa famille mais cette année-là, après deux années passées en Angleterre avec ses quatre enfants, il établit sa famille à Paris et continue de voyager seul à travers l'Europe. 


\section{Le système d'échange}

7 C'est alors que s'opère un tournant dans la carrière d'Alexandre. Ayant passé plusieurs années en Angleterre, aux Pays-Bas ou en Allemagne, ayant parcouru la Belgique, l'Allemagne, la Lettonie, la Hongrie, la Pologne, la Russie, l'Autriche, l'Italie, les ÉtatsUnis et le Canada, il observe les cours royales et les universités qu'il parcourt. Pendant ses voyages, il s'aperçoit du fait que beaucoup de bibliothèques ou musées ont des pièces d'art, des livres, des pièces de monnaie ou des médailles en plusieurs exemplaires. Il se met à inventorier ces listes et constitue une "bibliothèque de ressources internationales » qui incluait les fonds de Saint Petersbourg et de Vienne, elles-mêmes constituées de dizaines de milliers de pièces (Dargent, AF : 34). Il convainc alors les intellectuels et puissants d'échanger les doubles tout en leur garantissant ainsi l'enrichissement de leurs propres collections.

Nous sommes en 1825 et Alexandre a commencé alors une double carrière d'acteur et d'organisateur d'échanges, se servant de ses dons d'acteur pour diffuser l'idée des échanges internationaux. Il est convaincu de l'importance de la diffusion de la culture des nations et que celle-ci passe par les institutions. Mais, malgré l'enthousiasme des politiques et des grands de ce monde pour son œuvre, il a le plus grand mal à la financer. Il y consacre donc sa fortune fondant une Agence Européenne des Échanges qu'il veut durable et qu'il établit à Paris. Pour son agence, l'acteur ayant rassemblé nombre de dédicaces, fait paraitre aux Pays-Bas et en Angleterre un choix de lettres, d'attestations et d'autographes qu'il a reçus de ces personnages célèbres, dans un recueil illustré de peintures, de gravures et de lithographies le représentant sur les scènes de Berlin, jusqu'à Londres ou Edimbourg. Il comprend, entre autres, des signatures de Napoléon, Lafayette, Louis XIV, Louis XVI, Érasme, Cromwell, Washington, Goethe, Scott, Schiller, Byron et de beaucoup d'autres encore. Ce volume appelé "Album cosmopolite ou Choix des collections de Monsieur Alexandre Vattemare » et qu'il dédie « aux artistes de tous les pays » lui permet de commencer à financer son agence d'échanges à laquelle il attribue le qualificatif d'Européenne. Il est intéressant de voir qu'Alexandre Vattemare insiste déjà sur une diffusion de la culture qui n'est pas freinée par les frontières culturelles. Le succès de cet ouvrage est considérable et il participe largement à la propagation de ses idées sur la diffusion du patrimoine mondial (Vattemare, Album Cosmopolite : 2).

9 Il est remarquable de constater que l'échange et la promotion de ses idées sont nés d'une démarche de rencontre et de connaissance approfondie des peuples rencontrés. Dès 1831, la presse berlinoise souligne cette nouvelle orientation de sa carrière. En 1832, les Pays-Bas où il passe à nouveau six mois, l'enthousiasment. C'est à Hanovre en 1834 qu'est mentionné pour la première fois « l'Institut Européen des Échanges » établi à Paris (Dargent, AF : 69). Cette annonce sera bientôt suivie par beaucoup d'autres. Peu à peu, l'organisateur des échanges prend le pas sur l'acteur de génie. Pourtant déjà noble ambassadeur de la langue et de la culture française, la France reste son partenaire le plus difficile à convaincre.

\section{Vers le partage du patrimoine mondial}

10 Force est de constater que la foi dans l'interculturel d'Alexandre Vattemare est née d'abord de ses séjours prolongés à l'étranger: plusieurs années en Angleterre, une 
année aux Pays-Bas, en Allemagne, plusieurs mois en Hongrie, Tchécoslovaquie, Russie, Belgique et des voyages longs l'obligeant à faire de nombreuses étapes. Dépassant ce que l'on appelle souvent la barrière de la langue, il s'ancre dans les cultures locales. Son but est de mieux connaître les habitants pour mieux les séduire, rencontres déjà fondées sur une démarche d'échange, une sorte de "Dis-moi qui tu es et je te ferai rire ». En Russie, il adapte le titre de sa pièce «Les ruses de Nicolas » afin d'éviter d'offenser le tsar. L'approfondissement de ses compétences linguistiques, pragmatiques, et stratégiques lui permet de se faire l'ambassadeur de la langue et de la culture française sans s'y arrêter comme en témoignent les nombreuses marques de soutien et de gratitude qu'il recevra par la suite. À titre d'exemple, il reçoit en 1852, une lettre du ministre de l'intérieur des Pays-Bas, J. R. Thorbecke, le nommant, au nom du roi, agent pour la Hollande des échanges participant ainsi aux «trésors de la civilisation» (Vorstman-Le Pichon, AF : 108). La société de littérature néerlandaise (Maatschappij der Nederlandsche Letterkunde) de Leiden lui offre le titre de membre. Malgré les Guerres européennes, il continue d'établir «l'Agence Européenne des Échanges » montrant que l'échange intellectuel dépasse les frontières politiques.

11 Mais une autre étape se dessine dans sa vie. En 1839, se présente pour Alexandre Vattemare, la possibilité de visiter l'Amérique du Nord, voyage prévu pour trois mois mais qui durera finalement deux années. Pour comprendre ce qui est remarquable dans cette entreprise de Vattemare en Amérique du Nord, il est important de se replacer dans la vision que les Français avaient à l'époque des Américains, encore forte des stéréotypes «bons sauvages [...] où vit un peuple sans culture " (Harter 2007 : 196). Il faut la vision de Vattemare pour y voir au contraire un promoteur de son système universel d'échanges par les institutions. En effet, à la même époque, on discute aux États-Unis, de l'avenir de l'héritage laissé par James Smithson qui souhaitait créer une institution pour l'augmentation et la diffusion du savoir parmi les hommes. On voit dès lors l'intérêt qu'Alexandre Vattemare peut y porter, puisqu'il a une même vision du partage de la culture. Pour lui, l'objet à valeur artistique ou scientifique doit être rendu accessible au public comme en témoigne cet extrait d'un article qu'il écrit, paru dans un journal anglais : "Si un homme cache et enfouit son or, on l'accuse et avec justice; il fait un vol au travail, il enlève un capital à la circulation, qui le multiplie et le féconde " (Nash 2007 : 159). Vattemare, voyageur infatigable, à défaut de convaincre la France, entreprend alors de visiter plus de cinquante villes d'Amérique du Nord, de la Nouvelle Orléans à Montréal en passant par Boston, Washington et New-York et s'appuie sur une constitution américaine forte pour promouvoir son système. Il n'arrive pas les mains vides. Il apporte, a chacun de ses voyages, des doubles des œuvres, livres, pièces de monnaie ou objets d'art qu'il distribue. Ainsi, il convainc les Américains d'ouvrir des bibliothèques, des musées et de centraliser leurs échanges (Deschamps 2007 : 187). Il contribue à la fondation des bibliothèques de Boston et de New-York et se voit confier par le congrès les échanges américains de la "Smithsonian Institution ». Le maire de Boston, Joseph Quincy, lui dédie son discours d'inauguration de la bibliothèque, le qualifiant de «bienfaiteur de l'humanité » (Le Pichon, J. AF : 23). De retour à Paris, il distribue les pièces rapportées parmi les différentes institutions. Son slogan est à l'époque «donner dans la joie, recevoir avec gratitude » (Vattemarre $1850: 7$ ), slogan qu'il proclame tout en se plaignant des Français qui reçoivent plus volontiers qu'ils ne donnent. Alors il s'appuie sur son principal allié, l'Amérique du nord. 
12 De retour aux États-Unis en 1848, il obtient l'accord de nombreux états et du congrès pour son œuvre d'échanges. Mais il va plus loin. Lui qui s'était imprégné des mœurs et des usages des populations qu'il rencontrait, décide de recevoir à Paris des indiens Iowas arrivés avec le peintre Catlin, s'intéressant à leur regard sur la société française de l'époque (Le Pichon, A. $2007: 225$ ). Il organise pour eux des rencontres qui seront plus tard relatées par ce même Catlin (Catlin 1848: 178-203). Il leur fait visiter les musées et illustres institutions, arrange une rencontre avec le roi et avec les savants français. La première rencontre se passe remarquablement bien et les Indiens lui en sont reconnaissants.

13 Étrangement, c'est la rencontre avec les savants qui sera un échec complet et qui peut le mieux nous éclairer sur le rôle que peut jouer la médiation dans la rencontre interculturelle. Cette rencontre a lieu à l'académie royale des sciences. Il est intéressant de lire le récit de Catlin à ce sujet. Curieux et avides de connaissances, les académiciens dissèquent des yeux leurs hôtes indiens, mesurant et palpant les différents membres de leur corps, observant leurs démarches, mais le tout, sans même leur offrir une chaise pour s'asseoir (Catlin 1848: 225). Ces éminents savants ont eux aussi, le désir de connaître leurs hôtes mais ils ne sont pas dans une démarche de rencontre. S'interrogeant sur le bien fondé de cette visite, le groupe d'Indiens Iowas questionna Vattemare à ce propos. Vattemare dans un souci de diplomatie, voulant excuser la démarche de ses compatriotes, leur décrit ces savants comme étant des «medicine men ». Bien loin de rassurer ses hôtes, cela eut pour effet de les affoler, persuadés qu'ils étaient d'avoir perçu dans leurs regards un appétit de dissection. Ils exprimèrent alors le désir de ne jamais les revoir et n'eurent, à partir de ce moment-là et malgré l'impression favorable qu'ils avaient eu du roi, qu'une idée : quitter la France (Catlin 1848 : 227).

14 Cette visite est pour nous instructive car elle nous montre que vouloir connaître l'autre n'implique pas forcément la rencontre. Décrire dans une démarche interculturelle et pragmatique les cultures, faire des analyses discursives, sociales, imagologiques, ethnologiques ou anthropologiques et accumuler les connaissances, devenir savant et expert en interculturel, voyager dans ce but et dans le but de promouvoir sa langue ou sa culture est une démarche vaine et même dangereuse, si elle n'est pas accompagnée d'un véritable désir de rencontre et d'échange. Mais ce désir ne suffit pas: la vie d'Alexandre Vattemare est instructive à plus d'un titre. Elle nous montre que la rencontre passe par un séjour prolongé chez l'autre, accompagné d'une démarche d'apprentissage de la langue, d'un approfondissement de la connaissance des coutumes et usages et que cette connaissance en réduisant la peur de l'inconnu, en faisant tomber les barrières, conduit inexorablement à une certaine amitié avec la personne ou le peuple rencontré. Cette amitié s'exprimera plus tard pour Vattemare ayant pris conscience du drame de l'extinction des langues et cultures indigènes en Amérique du Nord. Un chef indien lui demandera son intervention et il en prendra la défense dans un discours (désespéré ?) au Parlement canadien.

\section{Conclusion}

15 À partir des différents ouvrages et documents témoins de son activité, nous avons vu comment d'un étudiant en médecine qui faisait parler les cadavres, Alexandre Vattemare est devenu par l'ambassade de la langue et de la culture françaises, un 
défenseur du patrimoine culturel international, établissant des rapports étroits entre les notions de langues, identités et cultures. Alexandre Vattemare, par l'échange, passe d'une communication interculturelle à une communication transculturelle, échanges de savoirs fondés sur l'amitié et le respect de celui avec qui l'on communique. Sans évincer les processus sociaux, linguistiques et cognitifs essentiels dans la démarche interculturelle, c'est-à-dire l'accumulation d'expériences, d'interactions permettant l'acquisition de connaissances et de la réflexion qui s'ensuit, la vie d'Alexandre Vattemare montre que la connaissance des différentes composantes des identités ethniques, sociales ou encore nationales, en tant que systèmes de valeurs plus ou moins normés, ne suffit pas. Son parcours met en avant le fait qu'on ne peut se passer d'un engagement né d'une expérience de vie commune, d'un don de soi. Pourtant, malgré les soutiens illustres, la Guerre de sécession aux États-Unis, en mettant fin aux financements de son agence, met un frein à l'épanouissement de son système d'échanges et Alexandre Vattemare y investit le reste de sa fortune. En 1864, il meurt dit-on, épuisé, après avoir écrit une lettre désespérée à Guizot et restée sans réponse. La formidable collection accumulée par cet acteur philanthrope de génie est vendue aux différents musées. Plus de " 6000 objets de valeur scientifique et esthétique " contribuent à l'entretien ou à la création de musées aux États-Unis ou en Europe. Mais l'Agence Européenne des Échanges internationaux s'éteint. Pourtant, Alexandre Vattemare a jeté les bases d'institutions d'échanges culturels. En plein siècle de bouleversements majeurs, où l'esprit de colonisation se répandait, moins occupé par l'idée de l'expansion d'une langue et d'une culture, Alexandre Vattemare nous a montré que la conservation du patrimoine international se fonde avant tout sur la connaissance réciproque. Cette connaissance réciproque est fondée sur l'écoute et le temps passé à se rencontrer, l'échange gratuit, l'amitié et le respect qui en découlent. Des notions de diffusion et de défense des langues et des cultures, concepts aux résonnances colonisatrices ou guerrières, il me semble qu'il est temps de glisser vers des notions de don et d'échange. Ainsi, témoignant par là-même de notre propre identité toujours mouvante, nous entrerons dans une démarche de construction mutuelle. Alexandre Vattemare reprochait aux Français de recevoir avec joie mais de donner avec peine, qu'en est-il aujourd'hui ?

\section{BIBLIOGRAPHIE}

BRUNEAU, C. (1948). Histoire de la langue française des origines à nos jours. t. XII. L'époque romantique. Armand Colin. Paris.

CATLIN, G. (1848). Catlin's notes : eight years travel and residence in Europe, with his North American Indian collection. Vol. II, London.

http://books.google.nl/books?

id=GoZIAAAAMAAJ\&printsec=frontcover\&hl=fr\&source=gbs_ge_summary_r\&cad=0 -

$\mathrm{v}=$ onepage \&q\&f=false 
DARGENT, J.L. (archives familiales). « Alexandre Vattemare, 7 novembre 1796 - 7 avril 1864. Fondateur de l'Agence Européenne des Échanges internationaux ». In Patrick Manificat. Monsieur Alexandre, ventriloque et philanthrope (1796-1864). Documents, correspondance et illustrations.

DESCHAMPS, M. (2007). « Au cœur du système Vattemare : l'Agence des échanges internationaux ». In P-A Tiliette \& E. Havens (dir.). L'Ambassadeur extravagant. Alexandre Vattemare, ventriloque et pionnier des échanges culturels internationaux. Boston Public Library \& Paris Bibliothèque. Le Passage, 169-178.

GWINN, N.E. (2007). « Vattemare et la Smithsonian Institution ». In L'Ambassadeur extravagant. Alexandre Vattemare, ventriloque et pionnier des échanges culturels internationaux. Boston Public Library \& Paris Bibliothèque. Le Passage, 179-190.

HARTER, H. (2007). « L'exposition universelle de Paris de 1855 : un moment privilégié dans les relations entre Alexandre Vattemare et les Américains ». In P-A Tiliette \& E. Havens (dir.). L'Ambassadeur extravagant. Alexandre Vattemare, ventriloque et pionnier des échanges culturels internationaux. Boston Public Library \& Paris Bibliothèque. Le Passage, 191-200.

KRAMSCH, C. (2008). « Voix et contrevoix : l'expression de soi à travers la langue de l'autre ». In Geneviève Zarate, Danièle Lévy \& Claire Kramsch (dir.). Précis du plurilinguisme et $d u$ pluriculturalisme. Paris : Éditions des archives contemporaines, 35-38.

LE PICHON, A. (2007). «Vattemare et la connaissance réciproque ». In P-A. Tiliette \& E. Havens (dir.). L'Ambassadeur extravagant. Alexandre Vattemare, ventriloque et pionnier des échanges culturels internationaux. Boston Public Library \& Paris Bibliothèque. Le Passage, 216-217.

LE PICHON, J. (Archives familiales). « Alexandre Vattemare ». In Patrick Manificat. Monsieur Alexandre, ventriloque et philanthrope (1796-1864). Documents, correspondance et illustrations, 23-27.

LE PICHON-VORSTMAN, E. (Archives familiales). « Alexandre aux Pays-Bas ». In Patrick Manificat. Monsieur Alexandre, ventriloque et philanthrope (1796-1864). Documents, correspondance et illustrations, 107-108.

VATTEMARE, A. (Archives familiales) a. Extraits de l'Album cosmopolite. (1837). In Itinéraires dans plusieurs pays de l'Europe, Texte intégral transcrit et illustré par Patrick Manificat.

VATTEMARE, A. (Archives familiales) b. In Patrick Manificat. La revue de Presse (1830-1839)

d'Alexandre Vattemare.

VATTEMARE, A. (1850). Second report on international literary exchanges. Albany : Weed Parsons \& co. public printers. http://books.google.nl/books/about/

Description_of_a_Monument_to_the_Memory.html?id=vwj_cQAACAAJ\&redir_esc=y

\section{NOTES}

1. Les initiales AF sont utilisées pour désigner les différents recueils édités dans un cadre familial par Patrick Manificat et rassemblant diverses contributions. 


\section{RÉSUMÉS}

En 1864 disparaissait Alexandre Vattemare, tour à tour ventriloque et acteur célébré dans l'Europe entière, fondateur de l'Agence Européenne des Échanges Internationaux ou de la bibliothèque de Boston, inspirateur du Smithsonian Institute, devenu défenseur des minorités linguistiques et, paradoxalement, noble ambassadeur de la langue et de la culture françaises. Ami de Goethe, avec qui il conversait en français mêlé de mots d'allemands, salué par de nombreuses personnalités dont Thorbecke pour les Pays-Bas ou Walter Scott en Angleterre, pour être compris et apprécié, il jouait dans la langue du pays, en allemand en Allemagne et en Autriche, en anglais en Grande-Bretagne et aux États-Unis ; mais aux Pays-Bas, en Pologne, en Russie et en Italie, c'est en français qu'il s'exprimait. Fasciné par le patrimoine mondial, sa foi dans l'interculturel et sa conviction, que la mise en valeur des patrimoines linguistiques et culturels passe par les institutions, remportent alors un succès étonnant qui à l'heure d'internet et des bibliothèques digitales, des séjours Erasmus ou de l'expatriation plus ou moins temporaire, nous amène à réfléchir sur le sens de la diffusion des langues et des cultures et en particulier de la langue et de la culture françaises.

In 1864 disappeared Alexander Vattemare. Ventriloquist and actor celebrated throughout Europe, among others, he founded the European Agency for International Exchange, the Boston Public Library and inspired the Smithsonian Institute. He became a defender of linguistic minorities and a noble Ambassador of the French language and culture. Friend of Goethe, with whom he conversed in French and German and praised by numerous personalities including Thorbecke In The Netherlands or Walter Scott in England. To be better understood and appreciated, he performed in the language of the country; German in Germany and Austria, English in Britain and the United States, but mostly French in the Netherlands, Poland, Russia and Italy. He was fascinated by world heritage and his faith in intercultural values and beliefs that institutions must be at the heart of the spread of linguistic and cultural heritages were a surprising success. At the dawn of the Internet and of digital libraries, of Erasmus and temporary expatriation, reflecting upon his ideals and accomplishments leads us to think about the meaning of the dissemination of the languages and cultures, particularly of the French language and culture.

\section{INDEX}

Mots-clés : XIXe siècle, langue et culture françaises, échanges internationaux, institutions pour le partage des langues et des cultures

Keywords : institutions to promote the exchange of canguages and cultures, 19th Century, French language and culture, international exchanges

\section{AUTEUR}

\section{EMMANUELLE LE PICHON-VORSTMAN}

Université d'Utrecht

E.M.M.LePichon@uu.nl 